\title{
Safety and efficacy of intrathecal ziconotide in the management of severe chronic pain
}

REVIEW

This article was published in the following Dove Press journal:

Therapeutics and Clinical Risk Management

2 July 2009

Number of times this article has been viewed

\author{
Howard S Smith' \\ Timothy R Deer ${ }^{2}$ \\ 'Albany Medical College, Department \\ of Anesthesiology, Albany, New York, \\ USA; ${ }^{2}$ The Center for Pain Relief, \\ Clinical Professor, West Virginia \\ University, Charleston, West Virginia, \\ USA
}

Abstract: Ziconotide is a conopeptide intrathecal (IT) analgesic which is approved by the US Food and Drug Administration (FDA) for the management of severe chronic pain. It is a synthetic equivalent of a naturally occurring conopeptide found in the venom of the fish-eating marine cone snail and provides analgesia via binding to $\mathrm{N}$-type voltage-sensitive calcium channels in the spinal cord. As ziconotide is a peptide, it is expected to be completely degraded by endopeptidases and exopeptidases (Phase I hydrolytic enzymes) widely located throughout the body, and not by other Phase I biotransformation processes (including the cytochrome P450 system) or by Phase II conjugation reactions. Thus, IT administration, low plasma ziconotide concentrations, and metabolism by ubiquitous peptidases make metabolic interactions of other drugs with ziconotide unlikely. Side effects of ziconotide which tend to occur more commonly at higher doses may include: nausea, vomiting, confusion, postural hypotension, abnormal gait, urinary retention, nystagmus/amblyopia, drowsiness/somnolence (reduced level of consciousness), dizziness or lightheadedness, weakness, visual problems (eg, double vision), elevation of serum creatine kinase, or vestibular side effects. Initially, when ziconotide was first administered to human subjects, titration schedules were overly aggressive and led to an abundance of adverse effects. Subsequently, clinicians have gained appreciation for ziconotide's relatively narrow therapeutic window. With appropriate usage multiple studies have shown ziconotide to be a safe and effective intrathecal analgesic alone or in combination with other intrathecal analgesics. Keywords: pain, ziconotide, intrathecal analgesics, safety, patient acceptability

\section{Introduction}

The US Food and Drug Administration (FDA) approved ziconotide (Prialt ${ }^{\mathbb{R}}$; Elan Pharmaceuticals, Inc.) on December 28, 2004 for the management of severe chronic pain in patients whom intrathecal (IT) therapy is warranted, and who are intolerant of or refractory to other treatments, such as systemic analgesics, adjunctive therapies, or IT morphine. Ziconotide is approved for use only in the Medtronic SynchroMed $^{\circledR}$ EL, SynchroMed ${ }^{\circledR}$ II Infusion System, and the CADD-Micro ${ }^{\circledR}$ ambulatory infusion pump.

Prialt ${ }^{\circledR}$ contains ziconotide acetate, with L-methionine $(0.05 \mathrm{mg} / \mathrm{mL})$ and sodium chloride as excipients at $\mathrm{pH} 4.0$ to 5.0. Ziconotide is a synthetic equivalent of a naturally occurring conopeptide found in the venom of the fish-eating marine cone snail, Conus magnus. This peptide, formerly known as SNX-111 or $\omega$-conotoxin MVIIA, is a component of the venom used to immobilize the snail's prey. Ziconotide is a 25 amino acid, polybasic peptide containing 6 cysteine residues linked by 3 disulfide bridges with a molecular weight of $2639 \mathrm{Da}$ and a molecular formula of $\mathrm{C}_{102} \mathrm{H}_{172} \mathrm{~N}_{36} \mathrm{O}_{32} \mathrm{~S}_{7} \cdot{ }^{1}$
Correspondence: Howard S Smith Associate Professor and Academic Director of Pain Management, Albany Medical College, Department of Anesthesiology, 47 New Scotland Avenue; MC- I 3 I, Albany, New York 12208, USA

Tel + | 5l8-262-446|

Fax + I 518-262-267I

Email smithh@mail.amc.edu 
The amino acid sequence and disulfide bridging pattern are given below:

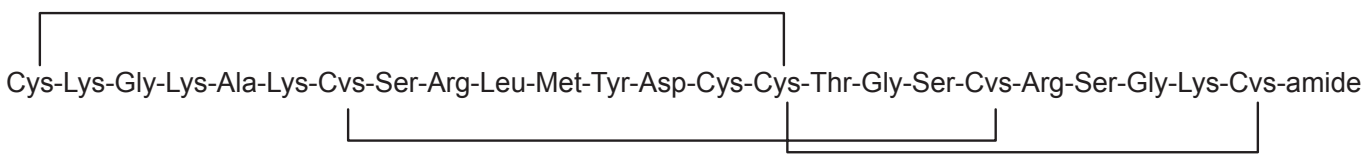

The three disulfide bridges contribute to stabilization, of ziconotide's well-defined three-dimensional structure, ${ }^{2-4}$ which is in part shaped as a cylinder. The cylinder represents the amide backbone of ziconotide VIIA overlayed against an electrostatic potential surface. Ziconotide is a hydrophilic molecule that is freely soluble in water and is practically insoluble in methyl t-butyl ether.

\section{Conopeptides}

The venoms of the $\sim 700$ species of predatory cone snails (genus Conus) are being systematically characterized. Each Conus species contains 100 to 200 small, highly structured venom peptides (colloquially known as conotoxins or conopeptides), which are synthesized and secreted in a venom duct. $^{5}$ Thus, living Conus are likely to express $>70,000$ different peptides in their venoms. ${ }^{6}$

Based on their disulfide connectivity, a highly conserved $\mathrm{N}$-terminal precursor sequence and a similar mode of action, conotoxins can be classified into 11 superfamilies: A, D, I1, I2, J, L, M, O, P, S, and T superfamily ${ }_{.}^{6,7}$ Each superfamily may possess different mechanisms of action and may also be divided into several families according to the cysteine arrangement. $^{8}$

Three analgesic targets of analgesic conopeptides include: N-type voltage-sensitive calcium channels ( $\omega$-ziconotide), $\omega$-conotoxin MVIIA also known as $\mathrm{N}$ methyl-d-aspartate [NMDA] receptors (conantokin-G), and neuronal voltage-gated sodium channels (mu-conotoxin KIIIA).

Teichert et al reported the discovery and characterization of three conantokin peptides from the venom of Conus parius. Each peptide (conantokin-Pr1, - $\operatorname{Pr} 2$, and -Pr3) contains 19 amino acids with 3 gamma-carboxyglutamate (Gla) residues, a post-translationally modified amino acid characteristic of conantokins. ${ }^{9}$ Electrophysiological assays confirmed that conantokins- $\operatorname{Pr} 1,-\operatorname{Pr} 2$, and $-\operatorname{Pr} 3$ are N-methyl-d-aspartate (NMDA) receptor antagonists, with highest potency for NR2B-containing NMDA receptors. ${ }^{9}$

mu-Conotoxin KIIIA, from Conus kinoshitai, blocks mammalian neuronal voltage-gated sodium channels (VGSCs) and is a potent analgesic following systemic administration in mice. ${ }^{10}$

\section{Mechanism of action}

Ziconotide binds to N-type voltage-sensitive calcium channels (NVSCCs) [Cav2.2] located on the primary nociceptive (A- $\delta$ and $C$ ) afferent nerves in the superficial layers (Rexed laminae I and II) of the dorsal horn in the spinal cord. Ziconotide produces potent antinociceptive effects ${ }^{11}$ by selectively binding to $\mathrm{N}$-type voltage sensitive calcium channels $\mathrm{s}^{12,13}$ on neuronal somata, dendrites, dendritic shafts, and axon terminals, thus blocking neurotransmission from primary nociceptive afferents. ${ }^{14}$ Complete chemical synthesis of ziconotide was achieved in 1987, and N-type voltagesensitive calcium channels (NVSCCs) were subsequently identified as its target site. ${ }^{12}$ It potently inhibits the conduction of nerve signals ( $K$ i value of $0.5 \mu \mathrm{M}$ ) ${ }^{15}$ by specifically blocking the NVSCC. In the complex with NVSCC, it forms a compact folded structure with a binding loop between Cys8 and Cys 15 that also contains Tyr13, an important amino-acid residue located at the binding site. ${ }^{16,17}$

NVSCCs are found exclusively in presynaptic neurons where they regulate depolarization-induced calcium influx, which subsequently control a variety of calcium-dependent processes. NVSCCs are abundantly present in the superficial lamina of the spinal-cord dorsal horn, where they have an important role in the spinal processing of nociceptive afferent (pain signaling) activity..$^{18,19}$ The potent inhibition and highly selective affinity of $\omega$-conotoxin MVIIA to NVSCC $(K d \text { value of } 9 \mathrm{pM})^{20}$ attracted interest to develop this peptide into an antinociceptive agent, since the inhibition of presynaptic NVSCCs results in blocking the release of presynaptic algesic neurotransmitters into the synapse.

Ziconotide may also bind to the delta opioid receptor; however, its affinity for the $\delta$-opioid receptor is 5 orders of magnitude lower than its affinity for NVSCCs. ${ }^{21}$

\section{Ziconotide preclinical evaluation}

Preliminary studies demonstrated that ziconotide has a remarkable potent antinociceptive profile in animal models with acute, persistent and neuropathic pain after intrathecal administration. ${ }^{21}$ In a rat incisional model of post-operative pain, ziconotide demonstrated more potent $\left(\mathrm{ED}_{50}\right.$ value of $49 \mathrm{pM})$ and longer activity than intrathecal morphine $\left(\mathrm{ED}_{50}\right.$ value of $2.1 \mathrm{nM}){ }^{22}$ 
Ziconotide displayed no mutagenic or carcinogenic potential and no propensity to induce cell proliferation or apoptosis in the surrounding CNS tissues. Local toxicity consisted of spinal cord compression with associated chronic inflammation attributed to pressure exerted by the IT catheter in control and ziconotide treated rats and dogs. Dogs treated with IT ziconotide displayed no evidence of granuloma formation at the catheter tip, ${ }^{23}$ as has been observed clinically with high-dose IT opioid therapy ${ }^{24}$ and in a canine IT infusion model at clinically relevant morphine doses. ${ }^{25}$ Ziconotide demonstrated no propensity to interfere with cardiac action potentials in vitro, induce QT prolongation in vivo, or deplete cardiac catecholamine levels at sympatholytic doses in rats. ${ }^{26}$ Although systemic anaphylaxis was observed in guinea pigs, the absence of antibodies to ziconotide in animals and human patients indicates that the drug has low immunogenic potential. ${ }^{26}$

Tolerance does not develop to the analgesia induced by intrathecal ziconotide in animal experiments and clinical trials. ${ }^{27-29}$ Furthermore, tolerance to morphine antinociception did not produce cross-tolerance to ziconotide and the antinociceptive effects of morphine and ziconotide are additive and sometimes even synergistic. Ziconotide did not produce respiratory depression in response to carbon dioxide. ${ }^{29,30}$

Nonclinical investigations of ziconotide included a comprehensive characterization of its toxicology, incorporating acute and subchronic toxicity studies in rats, dogs, and monkeys; reproductive toxicity assessments in rats and rabbits; and mutagenic, carcinogenic evaluations performed in vivo and in vitro. ${ }^{26}$ Additional investigations assessed the potential for cardiotoxicity (rats) and immunogenicity (mice, rats, and guinea pigs), and the presence or absence of intraspinal granuloma formation and local cell proliferation and apoptosis (dogs). ${ }^{26}$

\section{Pharmacokinetics}

The mean cerebrospinal fluid (CSF) volume of distribution for ziconotide is about $140 \mathrm{~mL}$ (range of 99.2-155 mL) after IT administration approximates the estimated total human CSF volume. ${ }^{31}$ Median CSF clearance (CL) of ziconotide $(0.38 \mathrm{~mL} / \mathrm{min})$ approximates the adult human CSF turnover rate, providing evidence that the primary mechanism for ziconotide CL is bulk CFS flow, rather than a metabolic process. The median elimination half-life of IT ziconotide from CSF in humans is roughly 4.6 hours (range of 2.9-6.5 hours) and protein binding is approximately $50 \% .{ }^{31}$ Although values are variable and dose dependent, following a 1 hour infusion of a 1 to $10 \mu \mathrm{g}$ dose, the area under the concentration-time curve (AUC) [a measure of total drug exposure that can be calculated (as the sum of trapezoids)] is in the range of 83.6 to
$608 \mathrm{ng} \cdot \mathrm{h} / \mathrm{mL}$ and the peak drug concentration $\left(\mathrm{C}_{\max }\right)$ is in the range of 16.4 to $132 \mathrm{ng} / \mathrm{mL}$. ${ }^{31}$

IT ziconotide is transported into the systemic circulation, where it may become bound to plasma proteins or degraded by proteolytic enzymes (the half-life of ziconotide in serum is 1.3 hours). ${ }^{32}$ Although formal pharmacokinetic (PK) drug-drug interaction studies have not been conducted with ziconotide, PK drug interactions are unlikely, due to ziconotide's low protein binding and low plasma concentrations following IT administration. ${ }^{32}$ Coadministration of ziconotide with other drugs that act on the CNS may have an additive effect. There is no need for dose reduction in response to renal or hepatic failure. ${ }^{32}$

The time course of ziconotide's observable pharmacologic action appears to develop more slowly than the kinetics of the distribution of ziconotide into the CSF. The median time to onset of the most commonly reported adverse events (AEs) ranged from 3 to 9.5 days. However, an approximation to steady-state CSF concentration generally is achieved within 24 hours (approximately five half-lives). In the Rauck et al study of 2006, dose changes could be made two to three times per week and no more frequently than every 24 hours. The relatively slow onset of AEs suggests that increments in the dosing of ziconotide be made no more frequently than weekly. ${ }^{33}$

The apparent lag between CSF pharmacokinetics and the pharmacodynamics of ziconotide may reflect the drug's slow penetration into the CNS parenchyma. Consistent with ziconotide's molecular weight of approximately $2500 \mathrm{Da}$ and its polycationic nature, microdialysis studies in rat brain with radioiodinated ziconotide have shown that no detectable ziconotide diffused more than $1 \mathrm{~mm}$ from the dialysis probe in 2 hours of perfusion. ${ }^{34}$ The slow diffusion of ziconotide in neural tissue may also explain the slower-than-expected time course of resolution of AEs with discontinuation of ziconotide therapy. As stated above, based on the 4.6-hour CSF halflife of the drug, pharmacologically active concentrations of ziconotide would be expected to be cleared within 24 hours.

The CSF PK of ziconotide have been studied after 1-hour IT infusions of 1 to $10 \mu \mathrm{g}$ of ziconotide to patients with chronic pain. ${ }^{31}$ Following one-hour IT administration of 1 to $10 \mu \mathrm{g}$ of ziconotide, both total exposure (AUC; range: $83.6-608 \mathrm{ng} \cdot \mathrm{h} / \mathrm{mL})$ and peak exposure $\left(\mathrm{C}_{\max }\right.$; range: $16.4-132 \mathrm{ng} / \mathrm{mL}$ ) values in the CSF were variable and dosedependent, but appeared approximately dose-proportional. ${ }^{31}$ During 5 or 6 days of continuous IT infusions of ziconotide at infusion rates ranging from 0.1 to $7.0 \mu \mathrm{g} /$ hour in patients with chronic pain, plasma ziconotide levels could not be quantified in $56 \%$ of patients using an assay with a lower limit of detection of approximately $0.04 \mathrm{ng} / \mathrm{mL} .{ }^{31}$ Predictably, patients 
requiring higher IT infusion dose rates were more likely to have quantifiable ziconotide levels in plasma.

\section{Distribution}

Ziconotide has a mean CSF volume of distribution (Vd) following IT administration that approximates the estimated total CSF volume $(140 \mathrm{~mL})$, and is roughly $50 \%$ bound to plasma proteins. ${ }^{35}$

\section{Metabolism}

Ziconotide is cleaved by endopeptidases and exopeptidases at multiple sites on the peptide. ${ }^{35}$ Some ziconotide will leave the CSF and gain access into the systemic circulation, where it would be susceptible to proteolytic cleavage by various ubiquitous peptidases/proteases present in most organs. It appears that ziconotide is readily degraded to peptide fragments and their individual constituent free amino acids in vivo. Peptide fragments resulting from proteolytic degradation of ziconotide have not been evaluated for biologic activity.

\section{Elimination}

Minimal amounts of ziconotide $(>1 \%)$ were recovered in the urine following intravenous ziconotide infustion. ${ }^{35}$ The terminal half-life of ziconotide in CSF after IT administration was about 4.6 hours (range 2.9-6.5 hours). Mean CSF clearance $(\mathrm{CL})$ of ziconotide approximates adult human CSF turnover rate $(0.3-0.4 \mathrm{~mL} / \mathrm{min}){ }^{35}$

\section{Drug interactions}

Formal PK drug-drug interaction studies have not been performed with ziconotide. ${ }^{35}$ As ziconotide is a peptide, it is expected to be completely degraded by endopeptidases and exopeptidases (Phase I hydrolytic enzymes) widely located throughout the body, and not by other Phase I biotransformation processes (including the cytochrome P450 system) or by Phase II conjugation reactions. ${ }^{35}$ IT ziconotide administration results in low plasma ziconotide concentrations, and metabolism by ubiquitous peptidases make metabolic interactions of other drugs with ziconotide unlikely. Also, since ziconotide is not highly bound in plasma (approximately 50\%) and is present only low plasma levels following IT administration, clinically relevant plasma protein displacement reactions involving ziconotide and co-administered medications are unlikely. ${ }^{35}$

\section{Interaction with opioids}

Ziconotide does not bind to the mu opioid receptor and its pharmacological effects are not blocked by opioid antagonists. ${ }^{35}$ In animal models, IT ziconotide potentiated opioid-induced reduction in gastrointestinal (GI) motility, but did not potentiate morphine-induced respiratory depression. ${ }^{35}$ It may be conceivable that certain neurotransmitters released from voltage-sensitive calcium channels contribute to normal GI motility. In rats receiving IT ziconotide, additive analgesic effects were observed with concurrent administration of morphine, baclofen, or clonidine. ${ }^{35}$ Concurrent administration of IT ziconotide and morphine did not prevent the development of morphine tolerance in rats. ${ }^{35}$

\section{Interaction with CNS depressants}

Of the 1254 patients treated, most received several concomitant systemic medications, including antidepressants (66\%), anxiolytics (52\%), antiepileptic drugs (47\%), neuroleptics (46\%), and sedatives (34\%). ${ }^{35}$ The use of systemic CNS depressants concomitant with IT ziconotide may be associated with an increased incidence of CNS adverse events such as dizziness and confusion. ${ }^{35}$

\section{Administration}

Ziconotide should be administered IT (not intravenously) by or under the direction of a physician experienced in the technique of IT administration and who is familiar with the drug and device labeling. Ziconotide is intended for IT delivery using a programmable implanted variable-rate microinfusion device or an external microinfusion device and catheter. ${ }^{35}$

Ziconotide is supplied as a $25 \mu \mathrm{g} / \mathrm{mL}$ solution in a single-use $20 \mathrm{~mL}$ glass vial and as a $100 \mu \mathrm{g} / \mathrm{mL}$ solution in single-use glass vials containing $1 \mathrm{~mL}, 2 \mathrm{~mL}$, or $5 \mathrm{~mL}$ of solution. Ziconotide is used for therapy undiluted $(25 \mu \mathrm{g} / \mathrm{mL}$ in $20 \mathrm{~mL}$ vial) or diluted $(100 \mu \mathrm{g} / \mathrm{mL}$ in 1,2 , or $5 \mathrm{~mL}$ vials $){ }^{35}$ Only the undiluted $25 \mu \mathrm{g} / \mathrm{mL}$ formulation should be used for ziconotide naïve pump priming. Diluted ziconotide is prepared with sterile preservative-free $0.9 \%$ sodium chloride injection USP, using aseptic procedures to the desired concentration prior to placement in the microinfusion pump. The $100 \mu \mathrm{g} / \mathrm{mL}$ formulation may be administered undiluted once an appropriate dose has been established. All ziconotide solutions should be refrigerated after preparation and infusion should be started within 24 hours. ${ }^{35}$ Due to adsorption of ziconotide to the titanium of the implanted pump and the dilution errors that occur with residual pump volume, despite reservoir evacuation, the rinse process helps to insure the concentration of the anticipated final pump solution. ${ }^{36}$ Therefore, it is very important that you do not initiate ziconotide without the appropriate rinse process as suggested by the manufacturer. ${ }^{35}$

Treatment should generally be initiated at a delivery of 0.001 to $0.05 \mu \mathrm{g} /$ hour and upwards titration should proceed 
extremely slowly. IT ziconotide should not be initiated at more than $2.4 \mu \mathrm{g} /$ day $(0.1 \mu \mathrm{g} /$ hour $)$. Doses may be titrated upward by 0.01 to $0.05 \mu \mathrm{g} /$ hour with a maximum increase of $2.4 \mu \mathrm{g} /$ day $(0.1 \mu \mathrm{g} /$ hour $)$ and at intervals of once per week but absolutely no more than 2 to 3 times per week, up to a recommended maximum of $19.2 \mu \mathrm{g} /$ day $(0.8 \mu \mathrm{g} /$ hour $)$ after 3 weeks. ${ }^{35} \mathrm{In}$ clinical trials, no rebound or other adverse events were related to discontinuation of ziconotide despite the fact that treatment was almost always discontinued abruptly. ${ }^{35}$

The dose of IT ziconotide should be slowly adjusted according to the patient's severity of pain, their response to therapy, and the occurrence of adverse events. The effective dose of ziconotide for analgesia is variable. The average dose level at the end of a 3-week titration period used in the slow titration clinical trial was $6.9 \mu \mathrm{g} /$ day $(0.29 \mu \mathrm{g} /$ hour $)$ and the maximum dose was $19.2 \mu \mathrm{g}$ /day $(0.8 \mu \mathrm{g} /$ hour $)$ on Day $21 .{ }^{35}$

An expert consensus statement, developed after the drug was marketed, recommends a starting dose of not more than $0.5 \mu \mathrm{g} / 24$ hours $(0.02 \mu \mathrm{g} /$ hour $)$ intrathecally and a slow titration schedule with potential interval increases of not more than $0.5 \mu \mathrm{g} / 24$ hours $(0.02 \mu \mathrm{g} /$ hour $)$ intrathecally once weekly. ${ }^{36}$ A slower starting rate and less frequent titration schedule may decrease the likelihood of serious adverse effects. Faster titration schedules should be considered only if there is an urgent need for analgesia and potential benefits outweigh risks to patient safety, in instances such as palliative care. However, clinicians should be aware that, when starting at faster infusion rates, adverse effects may require temporarily withholding the medication, thus delaying pain relief for the patient. ${ }^{36}$ Additionally, clinicians should be aware that many of the observed side effects of IT ziconotide cannot be prevented by slow titration; however, it is possible that their severity and frequency of adverse effects may be minimized by extremely slow and careful titration schedules.

\section{Contraindications and adverse effects}

The safety of IT ziconotide administered as a continuous infusion has been evaluated in 1254 patients participating in acute and severe chronic pain trials. The duration of treatment has ranged from a one-hour IT infusion to treatment lasting for more than 7.5 years. ${ }^{35}$ The mean duration of treatment was 193 days with 173 patients (14\%) treated for at least 1 year. The average final dose was $17.6 \mu \mathrm{g} /$ day $(0.73 \mu \mathrm{g} /$ hour $)$. The most frequently reported adverse events $(25 \%)$ in the 1254 patients (662 patient years) in clinical trials were dizziness, nausea, confusion, headache, somnolence, nystagmus, asthenia, and pain. ${ }^{35}$
Significant adverse events are less apt to occur when the drug is slowly titrated gradually over 3 weeks or longer.

Possible side effects of ziconotide may include:

- an allergic reaction,

- nausea, vomiting, seizures, fever, headache, and/or stiff neck (eg, meningitis),

- a change in mental status (cognitive and neuropsychiatric alterations) (extreme tiredness, asthenia, confusion, disorientation or decreased alertness),

- a change in mood or perception (hallucinations, unusual feelings in the mouth),

- postural hypotension, abnormal gait, urinary retention, nystagmus/amblyopia

- drowsiness/somnolence (reduced level of consciousness),

- dizziness or lightheadedness, weakness,

- visual problems (eg, double vision),

- elevation of serum creatine kinase, or

- vestibular side effects.

Vestibular side effects may be due to ziconotide blocking N-type calcium channels in the granular cell layer of the cerebellum. $^{37}$

Ziconotide is contraindicated in patients with a known hypersensitivity to ziconotide or any of its formulation components and in patients with any other concomitant treatment or medical condition that would render IT administration hazardous. Contraindications to the use of IT analgesia include: conditions such as the presence of infection at the microinfusion injection site, systematic infection with bacteremia, uncontrolled bleeding diathesis, and spinal canal obstruction that impairs circulation of CSF.

\section{Meningitis and other infections}

Meningitis can occur due to inadvertent contamination of the microinfusion device and other means such as CSF seeding due to hematogenous or direct spread from an infected pump pocket or catheter tract despite the use of strict aseptic techniques. While meningitis is rare with an internal microinfusion device and surgically-implanted catheter, the incidence increases substantially with external devices. In the 1254 patients in ziconotide clinical trials with an exposure of 662 patient-years, meningitis occurred at 3\% (40 cases) in the ziconotide group using either internal or external microinfusion devices and 1\% (1 case) in the placebo group with an exposure of only 5 patient-years. ${ }^{35}$ The risk of meningitis with external microinfusion devices and catheters was higher with $93 \%$ cases (38/41) occurring with external infusion systems. ${ }^{35}$ Serious infection or meningitis can occur within 24 hours of a breach in sterility such as a disconnected catheter. ${ }^{35}$ 


\section{Cognitive and neuropsychiatric adverse events}

CNS-related adverse events may occur with ziconotide, including psychiatric symptoms, cognitive impairment, and decreased alertness/unresponsiveness. For the 1254 patients treated, the following cognitive adverse event rates were reported: confusion (33\%), memory impairment (22\%), speech disorder (14\%), aphasia (12\%), thinking abnormal (8\%), and amnesia (1\%). ${ }^{35}$ Cognitive impairment may appear gradually after several weeks of treatment. The ziconotide dose should be reduced or discontinued if signs or symptoms of cognitive impairment develop, however, other contributing causes should be entertained or investigated as appropriate. Cognitive effects of ziconotide are usually reversible within 2 weeks (3-15 days) after cessation of therapy. ${ }^{35}$ Older persons are at higher risk for confusion. In placebo-controlled trials, there was a higher incidence of suicide, suicide attempts, and suicide ideations in ziconotide-treated patients $(\mathrm{N}=3)$ than in the placebo group $(\mathrm{N}=1) .{ }^{35}$ The incidence was 0.10 /patient year for placebo patients and $0.27 /$ patient-year for ziconotide patients. ${ }^{35}$

Acute psychiatric disturbances such as hallucinations $(12 \%)$, paranoid reactions $(3 \%)$, hostility $(2 \%)$, delirium $(2 \%)$, psychosis $(1 \%)$, and manic reactions $(0.4 \%)$ have been reported in patients treated with ziconotide. ${ }^{35}$ Patients with pretreatment psychiatric disorders may be at increased risks of the above reactions as well as the potential for IT ziconotide to facilitate or worsen depression with the risk of suicide in susceptible patients. Patients with pre-existing psychiatric disorders should be carefully evaluated before instituting ziconotide infusion as some of these patients (especially patients with a history of psychosis) may not be well suited for ziconotide therapy.

\section{Reduced level of consciousness}

Unresponsiveness has occurred while receiving ziconotide. The incidence of unresponsiveness or stupor in clinical trials was $2 \% .{ }^{35}$ If reduced levels of consciousness occur, ziconotide should be discontinued until the event resolves, and other etiologies (eg, meningitis) should be considered Patients taking concomitant antiepileptic drugs, neuroleptics, sedatives, or diuretics may be at higher risk to develop depressed levels of consciousness. ${ }^{35}$ If altered consciousness occurs, other central nervous system (CNS) depressant drugs should also be discontinued as clinically appropriate.

\section{Elevation of serum creatine kinase (CK-MM)}

Forty percent of patients from clinical studies (largely open label) had serum creatine kinase (CK) levels above the upper limit of normal, and $11 \%$ had CK levels that were $\geq 3 \mathrm{X}$ upper limit of normal (ULN) ${ }^{47}$ (only the muscle isoentyme (MM) was elevated in the cases where CK was fractionated). Although the CK elevations may occur at any time during therapy, the greatest incidence of CK elevation was during the first two months of treatment. ${ }^{35}$ Elevated CKs were more often seen in males, in patients who were being treated with anti-depressants or antiepileptics, and in patients treated with IT morphine. ${ }^{35}$ The majority of patients with elevations in $\mathrm{CK}$, even for prolonged periods of time, did not experience limiting side effects. One case of symptomatic myopathy with electromyography findings, and two cases of acute renal failure associated with rhabdomyolysis and extreme $\mathrm{CK}$ elevations (17,000-27,000 IU/L) have been reported. ${ }^{35}$

Prescribing information from the manufacturer recommends that physicians monitor serum $\mathrm{CK}$ in patients undergoing treatment with ziconotide periodically (eg, every other week for the first month and monthly as appropriate thereafter). ${ }^{35}$ During ziconotide infusions, consideration for reduction or discontinuation should be entertained in the setting of new neuromuscular symptoms (eg, myalgias, myasthenia, muscle cramps, asthenia/fatigue), weakness, persistent increasing CK levels, or a reduction in physical activity.

\section{Overdosage}

The maximum IT dose of ziconotide administered in clinical trials was $912 \mu \mathrm{g} /$ day which is $892.8 \mu \mathrm{g} /$ day over the maximum recommended IT ziconotide dose of $19.2 \mu \mathrm{g} /$ day. ${ }^{35}$ At IT doses greater than the maximum recommended dose, exaggerated pharmacological effects (eg, ataxia, nystagmus, dizziness, stupor, unresponsiveness, spinal myoclonus, confusion, sedation, hypotension, word-finding difficulties, garbled speech, nausea, and vomiting) may be observed, but respiratory depression did not occur. ${ }^{35}$ Overdoses may occur due to pump programming errors or incorrect drug concentration preparations. In these cases, patients were observed and ziconotide was either temporarily discontinued or permanently withdrawn. ${ }^{35}$ Most patients recovered within 24 hours after ziconotide discontinuation, at which point the ziconotide CSF concentration should be less than $5 \%$ of peak levels. ${ }^{35}$

Because ziconotide has no known "antidote", overdose is treated with general medical supportive measures as appropriate. ${ }^{35}$ There have been no reported cases of death with ziconotide overdose. ${ }^{35}$

\section{Clinical experience with ziconotide}

In efforts to assess the safety and efficacy of IT ziconotide in patients with cancer or AIDS who have refractory pain; Staats 
and colleagues ${ }^{14}$ performed a double-blind, placebo-controlled, randomized trial at 32 study centers in the United States, Australia, and the Netherlands. Intrathecal ziconotide was titrated over 5 to 6 days, followed by a 5 -day maintenance phase for responders and crossover of non-responders to the opposite treatment group. Mean percentage change in visual analog scale of pain intensity (VASPI) score from baseline to the end of the initial titration period. Of the population evaluated, 67 (98.5\%) of 68 patients receiving ziconotide and $38(95 \%)$ of 40 patients receiving placebo were taking opioids at baseline (median morphine equivalent dosage of $300 \mathrm{mg} /$ day for the ziconotide group and $600 \mathrm{mg}$ /day for the placebo group; $P=0.63$, based on mean values), and 36 had used intrathecal morphine. ${ }^{14}$ Mean (SD) VASPI scores were $73.6(1.8) \mathrm{mm}$ in the ziconotide group and $77.9(2.3) \mathrm{mm}$ in the placebo group $(P=0.18)$. Mean VASPI scores improved $53.1 \%$ (95\% confidence interval $[\mathrm{CI}], 44.0 \%-62.2 \%$ ) in the ziconotide group and $18.1 \%(95 \% \mathrm{CI}, 4.8 \%-31.4 \%)$ in the placebo group $(P<0.001)$, with no loss of efficacy of ziconotide in the maintenance phase. ${ }^{14}$ Pain relief was moderate to complete in $52.9 \%$ of patients in the ziconotide group compared with $17.5 \%$ in the placebo group $(P<0.001)$. Five patients receiving ziconotide achieved complete pain relief, and $50.0 \%$ of patients receiving ziconotide responded to therapy compared with $17.5 \%$ of those receiving placebo $(P=0.001) .{ }^{14}$ Intrathecal ziconotide provided clinically and statistically significant analgesia in patients with pain from cancer or AIDS. ${ }^{14}$ Compared with placebo, ziconotide was associated with a number of (typically doserelated) adverse events: abnormal gait, dizziness, nystagmus, confusion, somnolence, fever, postural hypotension, urinary retention, nausea, and vomiting. ${ }^{14}$

Patients (169 ziconotide, 86 placebo) with severe chronic nonmalignant pain unresponsive to conventional therapy and a VASPI score $\geq 50 \mathrm{~mm}$ were treated over a 6 -day period in an inpatient hospital setting. ${ }^{38}$ Initial starting dose was $0.4 \mu \mathrm{g} /$ hour and was titrated to analgesia or intolerance (maximum dose $7.0 \mu \mathrm{g}$ /hour). The starting and maximum doses were reduced to $0.1 \mu \mathrm{g} /$ hour and $2.4 \mu \mathrm{g} /$ hour, respectively, due to AEs. ${ }^{38}$

The mean percentage reduction in VASPI score from baseline was $31.2 \%$ and $6.0 \%$ for ziconotide- and placebotreated patients, respectively $(P \leq 0.001) .{ }^{38}$ During the initial titration phase, a significantly greater percentage of patients in the ziconotide group compared to the placebo group reported AEs, including abnormal gait, amblyopia, dizziness, nausea, nystagmus, pain, urinary retention, and vomiting. ${ }^{38}$

The most commonly reported SAEs among ziconotidetreated patients were dizziness (8 occurrences), confusion (6), urinary retention (4), nausea and/or vomiting (4), amblyopia or visual abnormalities (3), abnormal gait (3), stupor or somnolence (3), ataxia or vestibular disorders (3), and encephalopathy (2). ${ }^{38}$ Other nervous system SAEs reported included single cases of agitation, catatonic reaction, thinking abnormal, depression, and aphasia. ${ }^{38}$

The neurocognitive AEs, such as confusion, disorientation, memory impairment, speech difficulties, impaired concentration, mental slowing, or thought abnormalities, are the most troublesome of the ziconotide-related CNS AEs and, if not manageable by dose reduction, often required drug discontinuation..$^{38}$

There was a considerable incidence of ziconotide-associated AEs in the 2006 study of Wallace and colleagues ${ }^{38}$ due to the rapid titration and high doses administered. ${ }^{38}$ The narrow therapeutic window of IT ziconotide dictates a slower and more cautious titration, which subsequently became incorporated in the US product labeling. ${ }^{38}$

In an attempt to reduce side effects and increase tolerability, Rauck et al ${ }^{33}$ conducted a double-blind, placebocontrolled study using a slower titration schedule and lower maximum dose than previous studies in 220 patients with chronic, noncancer refractory to conventional treatment.

Patients randomized to ziconotide $(\mathrm{n}=112)$ or placebo $(\mathrm{n}=108)$ started IT infusion at $0.1 \mathrm{mg} /$ hour $(2.4 \mathrm{mg} /$ day $)$, increasing gradually $(0.05-0.1 \mathrm{mg} /$ hour increments $)$ over 3 weeks. The ziconotide mean dose at termination was $0.29 \mathrm{mg} /$ hour $(6.96 \mathrm{mg} /$ day $)$. Patients' baseline VASPI score was 80.7 (SD 15). Statistical significance was noted for VASPI mean percentage improvement, baseline to Week 3 (ziconotide [14.7\%] vs placebo [7.2\%; $P=0.036]$ ) and many of the secondary efficacy outcomes measures. Significant AEs reported in the ziconotide group were dizziness, confusion, ataxia, abnormal gait, and memory impairment. Discontinuation rates for AEs and serious AEs were comparable for both groups. Slow titration of ziconotide, a nonopioid analgesic, to a low maximum dose resulted in significant improvement in pain and was better tolerated than in two previous controlled trials that used a faster titration to a higher mean dose. However, compared with the two previous studies ${ }^{14,38}$ the current study had a higher incidence of AEs related to higher cortical functions, such as confusion and memory impairment.

Such differences could be attributable to the longer duration of the current study and the higher cumulative dose over three weeks compared to only 5 to 6 days in the two previous controlled trials. However, results from the MMSE in the current study indicated no substantial changes in mental 
status and no significant differences between the ziconotide and placebo groups.

However, no occurrences of respiratory depression, drug dependence, or withdrawal symptoms (potential hazards of opioid administration) were reported during this study, and there was no evidence of tolerance to ziconotide or of granuloma formation at the tip of the IT catheter (as is observed rarely during IT opioid treatment in association with neurological sequelae).

Overall, the results of this study suggest that IT ziconotide infusion therapy is an option for patients with severe, refractory chronic pain. Efficacy data from this study should be interpreted with caution, as there was no comparator group in the study. An important limitation of this study is that it is unlikely that rare AEs would have been detected. The detection of such AEs typically requires patient exposure well beyond that achieved in this study. ${ }^{39}$ Additionally, it has been suggested that open-label trials are not ideal for detecting and assessing the risk for rare AEs because of lack of a control group and the potential for under-reporting of treatment-emergent AEs. ${ }^{39}$ Thus far, AEs that have been commonly associated with ziconotide are generally not life-threatening and typically resolve upon discontinuation of therapy. In the case of a serious AE, ziconotide therapy can be abruptly interrupted or discontinued without concern regarding withdrawal effects.

Wallace et al conducted an open-label, multicenter study of patients with severe chronic pain who were followed after being initiated on intrathecal ziconotide infusions. ${ }^{40}$ Efficacy assessments included the Visual Analog Scale of Pain Intensity and Safety was assessed via adverse events (AEs), vital signs, and routine laboratory values. One-hundred and nineteen patients received ziconotide for $\geq 360$ days; total exposure was 350.9 patient years. Median duration of ziconotide therapy was 67.5 days (range, 1.2-1215.5 days); mean dose at last infusion was $8.4 \mu \mathrm{g} /$ day (range, $0.048-240.0 \mu \mathrm{g} /$ day). Median VASPI scores at baseline, month 1 , and the last available observation up to month 2 were $76 \mathrm{~mm}$ (range, 4-100 mm), $68 \mathrm{~mm}$ (range, 0-100 $\mathrm{mm}$ ), and $73 \mathrm{~mm}$ (range, 0-100 $\mathrm{mm}$ ), respectively. Most patients (99.7\%) experienced $\geq 1 \mathrm{AE}$. Most AEs were of mild (43.5\%) or moderate (42.3\%) severity; $58.6 \%$ of AEs were considered unrelated to ziconotide. The most commonly reported AEs ( $\geq 25 \%$ of patients) included nausea, dizziness, headache, confusion, pain, somnolence, and memory impairment. Clinically significant abnormalities ( $>3$ times the upper limit of normal) in creatine kinase levels were reported in $0.9 \%$ of patients at baseline, $5.7 \%$ at month 1 , and $3.4 \%$ at ziconotide discontinuation. No drug-related deaths, IT granulomas, or permanent adverse sequelae occurred with ziconotide therapy. Wallace et al concluded that IT ziconotide infusions are an option for patients with severe, refractory chronic pain. ${ }^{40}$

Webster et al performed an open-label multicenter study evaluating the safety and efficacy of intrathecal ziconotide infusions and included 78 patients with chronic pain who had completed one of two previous ziconotide clinical trials. ${ }^{41}$ Each patient's initial ziconotide dose was based on his or her dose from the study of origin and was adjusted as necessary on the basis of adverse events and analgesic effect. The median ziconotide dose was $6.48 \mu \mathrm{g} /$ day (range, $0.00-120.00 \mu \mathrm{g} /$ day) at the initial visit and ranged from 5.52 to $7.20 \mu \mathrm{g}$ /day across all study visits. The most commonly reported new adverse events that were considered ziconotide related were memory impairment (11.3\%); dizziness, nystagmus, and speech disorder $(8.5 \%$ each); nervousness and somnolence $(7.0 \%$ each); and abnormal gait (5.6\%). There was no evidence of increased adverse event incidence at higher cumulative ziconotide doses. Elevations in creatine kinase were noted, but the proportion of patients with creatine kinase elevations did not change from the initial visit to the termination visit (4.1\%each). Stable mean VASPI scores during the three years of the study suggested no evidence of increased pain intensity with increased duration of ziconotide exposure. Intrathecal ziconotide infusions appeared to be well tolerated and effectively maintained on a longer basis in patients whose response to ziconotide and ability to tolerate the drug had been previously demonstrated in the short-term. ${ }^{41}$

New AEs were reported in $91.0 \%$ of patients (71 of 78). A new severe AE was reported for $70.4 \%$ of these patients (50 of 71) at some time during the study. Thirty-seven of the 71 patients who experienced new AEs (52.1\%) reported at least one new AE that was considered ziconotide related. Eight of the 1044 new AEs reported during the study were considered to be both severe and related to ziconotide (ie, memory impairment, abnormal gait, chills, hostility, quadriplegia, and sweating increased). The most commonly reported new AEs that were considered by the investigators to be related to ziconotide include: abnormal gait $(5.6 \%)$, dizziness $(8.5 \%)$, memory impairment $(11.3 \%)$, nervousness (7.0\%), nystagmus $(8.5 \%)$, somnolence $(7.0 \%)$, and speech disorder $(8.5 \%)$. The incidence of reported new AEs was greater in younger patients (ie, <60 years; 97.9\%, [46 of 47]) than in older patients (ie, $>60$ years; $80.6 \%$, [25 of 31$]$ ). ${ }^{41}$

The most commonly ( $\geq 1 \%$ of patients) reported new serious adverse effects include: dehydration (11.4\%), lung disorder (14.3\%), pneumonia (17.1\%), and sepsis (14.3\%). ${ }^{41}$ New or ongoing adverse effects that occurred in this study 
leading to interruption or termination of ziconotide therapy include: quadriplegia, dry mouth, abdominal pain, infection, medication error, hypotension libido decrease, memory impairment (all 1.4\%), psychosis (2.7\%), catheter complications (4.1\%), and pump complications $(2.7 \%) .{ }^{41}$

Headache, insomnia, and nervousness were more common while patients were receiving $<4.800 \mu \mathrm{g} /$ day of ziconotide (difference $\geq 5$ patients); accidental injury, back pain, fungal infection, and hypokalemia were more common while patients were receiving $\geq 4.800 \mu \mathrm{g} /$ day of ziconotide (difference $\geq 5$ patients). There was no evidence of increased AE incidence at higher cumulative ziconotide doses.

Ziconotide exposure totaled 133.4 patient-years during this study ${ }^{41}$ and 339.2 patient-years when cumulative experience across this study of Webster et al $2008^{41}$ as well as the studies of origin were included.

In the 2000 and 2003 Polyanalgesic Consensus Conference guidelines, ${ }^{24,42}$ ziconotide was not assigned to a line in the treatment algorithm of intrathecal analgesics for pain relief because of limited experience with the drug and because of pending FDA review. However, in 2007, the Polyanalgesic Consensus Conference panel of experts based on relevant new literature and clinical experience decided that ziconotide should be "upgraded" to a "first-line IT analgesic" $" 43$ (see Table 1).

\section{Combinations of ziconotide and opioids}

To assess the safety and efficacy of adding intrathecal morphine to intrathecal ziconotide in patients treated with stable ziconotide doses; Webster et al conducted a multicenter, open-label study with a 4-week morphine titration phase during which ziconotide was held constant and an extension phase during which dosing of either drug could vary; in twenty-five patients with suboptimal pain relief receiving stable ziconotide doses ( $\geq 4.8 \mathrm{mcg} /$ day) in one of two ongoing ziconotide trials. ${ }^{44} \mathrm{Ziconotide}$ dosing remained constant during the titration phase; intrathecal morphine titration was based on each patient's daily systemic opioid dose at the study's start. During the extension phase, intrathecal ziconotide and morphine dosing were adjusted per investigator discretion.

Safety was assessed primarily via adverse events. Efficacy was analyzed via percentage change on the visual analog scale of pain intensity and in weekly systemic opioid consumption. Twenty-five patients enrolled. The most common ( $\geq 10 \%$ of patients in either study phase) study drug-related (ie, ziconotide/morphine combination [or ziconotide monotherapy in the extension phase only]) treatment-emergent adverse events (TEAEs) included dizziness, peripheral edema, pruritus, and nausea. The median IT morphine dose was $0.25 \mathrm{mg} /$ day at the initial visit and progressively increased during the titration phase, with median values of $0.25,0.50,1.00$, and $1.25 \mathrm{mg}$ /day at weeks $1,2,3$, and 4, respectively. During the extension phase, the median IT morphine dose was $1.50 \mathrm{mg}$ /day at week 8 and fluctuated from a high of $2.1 \mathrm{mg} /$ day at week 56 to a low of $0 \mathrm{mg} /$ day for each of the two patients who completed a week 72 visit. During the titration phase, two patients $(8.0 \%)$ reported 6 serious TEAEs, including accidental injury, ataxia, confusion, dehydration, subdural hematoma, and urinary tract infection, none of which were considered related to the study drug.

From the initial visit to week 4, visual analog scale of pain intensity scores improved by a mean of $26.3 \%$ (95\% CI, 15.6\%-37.1\%) but varied during the extension phase (mean percentage change from the initial visit ranged from $-0.4 \%$ at week 16 to $-35.0 \%$ at week 72 ). Thus, analysis of the primary efficacy variable showed a mean $26.3 \%$ reduction in VASPI score from the initial visit to week 4, and a supportive efficacy analysis using LOCF corroborated these findings. Reductions in pain intensity were seen as early as week 1 and progressively increased at weeks 2 and 3 as upward titration of IT morphine continued. As the majority of the patients in the study had previously received IT opioid therapy, it is unlikely that IT morphine was solely responsible for the VASPI score reductions noted during the study; VASPI score reductions were more likely attributable to IT ziconotide/morphine combination therapy. Mean percentage decrease in systemic opioid consumption from the initial visit was $49.1 \%$ at week 4 and $51.2 \%$ at week 56 of the extension phase. Webster and colleagues concluded that intrathecal morphine, combined with stable intrathecal ziconotide doses, reduced pain in patients with previously suboptimal pain relief on ziconotide monotherapy. ${ }^{44}$

Wallace et al also performed a study to assess the safety and efficacy of adding intrathecal ziconotide to intrathecal morphine in patients being treated with a stable intrathecal morphine dose. ${ }^{45}$ Wallace and colleagues conducted a phase II, multicenter, open-label study with a 5-week titration phase and an extension phase, enrolling 26 patients with suboptimal pain relief receiving stable intrathecal morphine doses (2-20 mg/day). Intrathecal morphine dosing remained constant during the titration phase. Ziconotide therapy began at $0.60 \mu \mathrm{g} /$ day and was titrated to a maximum of $7.2 \mu \mathrm{g} /$ day. During the extension phase, ziconotide and intrathecal morphine 
Table I Recommended algorithm for intrathecal polyanalgesic therapies, 2007

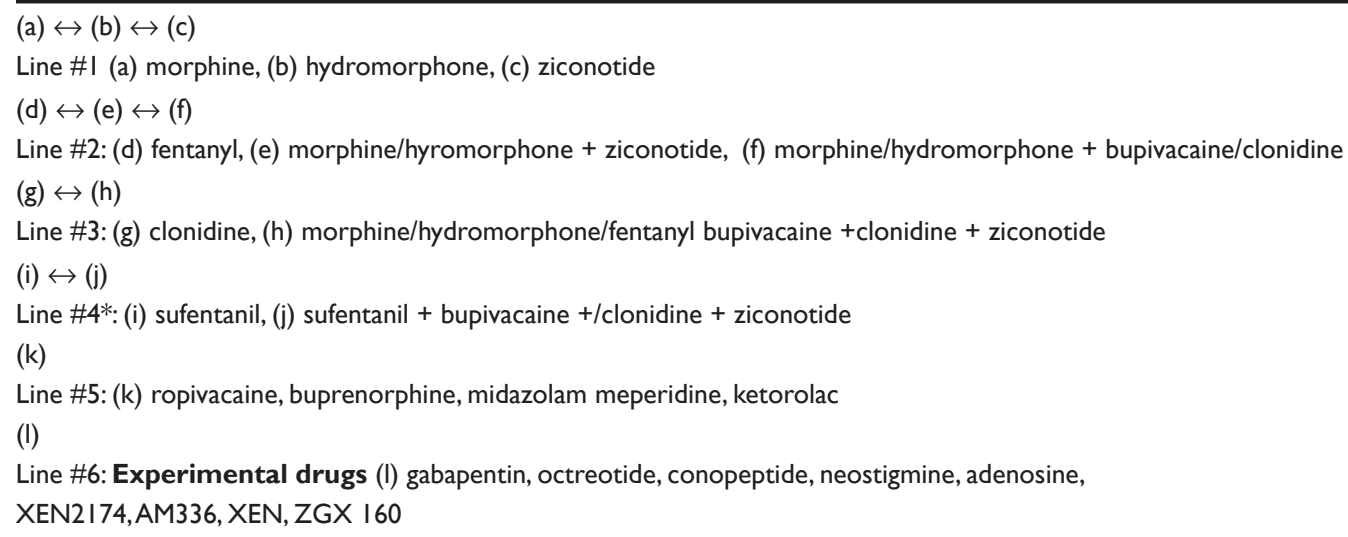

Notes: Line I:Morphine (a) and ziconotide (c) are approved by the US Food and Drug Administration for intrathecal analgesic use and are recommended for first-line therapy for nociceptive, mixed, and neuropathic pain. Hydromorphone (b) is recommended based on clinical widespread usage and apparent safety.

Line 2: Because of its apparent granuloma-sparing effect and because of its wide apparent use and identified safety, fentanyl (d) has been upgraded to a line 2 agent by the consensus conference when the use of the more hydrophilic agents of line I (a,b) result in intractable supraspinal side-effects. Combinations of opioid + ziconotide (e) or opioid + bupivacaine or clonidine ( $f$ ) are recommended for mixed and neuropathic pain and may be used interchangeably. When admixing opioids with ziconotide, attention must be made to the guidelines for admixing ziconotide with other agents.

Line 3: Clonidine $(\mathrm{g})$ alone or opioids such as morphine/hydromorphone/fentanyl with bupivacaine and/or clonidine mixed with ziconotide (h) may be used when agents in line 2 fail to provide analgesia or side-effects occur when these agents are used.

Line 4: Because of its proven safety in animals and humans and because of its apparent granuloma-sparing effects, sufentanil alone (i) or mixed with bupivacaine and/or clonidine plus ziconotide (j) is recommended in this line. The addition of clonidine, bupivacaine, and or ziconotide is to be used in patients with mixed or neuropathic pain. *In patients with end of life, the panelists felt that midazolam and octreotide should be tried when all other agents in lines I-4 have failed.

Line 5:These agents $(\mathrm{k})$, although not experimental, have little information about them in the literature and use is recommended with caution and obvious informed consent regarding the paucity of information regarding the safety and efficacy of their use.

Line 6: Experimental agents (I) must only be used experimentally and with appropriate Independent Review Board (IRB) approved protocols. ${ }^{32}$

dosing were adjusted at the investigator's discretion. ${ }^{45}$ In their study, treatment-emergent adverse events were generally mild or moderate; the most common ( $\geq 15 \%$ of patients in either study phase) study drug-related (ie, ziconotide/morphine combination [or ziconotide monotherapy in the extension phase only]) events were confusion, dizziness, abnormal gait, hallucinations, and anxiety. ${ }^{45}$ The mean percentage improvement in visual analog scale of pain intensity scores was $14.5 \%$ (95\% CI, $-9.4 \%$ to $38.5 \%$ ) from baseline to week 5 and varied during the extension phase (range: $-0.4 \%-42.8 \%$ ). Mean percentage change from baseline in systemic opioid consumption was $-14.3 \%$ at week 5 and varied considerably during the extension phase..$^{45}$

During the titration phase, 2 of 26 patients (7.7\%) reported 3 serious TEAEs, including cellulitis, cerebral ischemia, and stupor, none of which were considered related to the study drug. Cellulitis was considered related to intercurrent illness, whereas cerebral ischemia and stupor had unknown etiologies. During the extension phase, 4 of 18 patients (22.2\%) reported 9 serious TEAEs, including confusion, delusions, hallucinations, paranoid reaction (3 events in 3 patients), pneumonia, psychosis, and syncope. Most of these events $(6 / 9,66.7 \%)$ were considered unrelated to the study drug. The event of confusion was considered related to pneumonia; psychosis and one event of paranoid reaction were considered related to intercurrent illness; and one event of paranoid reaction, pneumonia, and syncope had unknown etiologies. One patient experienced delusions, hallucinations, and one episode of paranoid reaction that was considered related to the study drug.

When using combination IT therapy, drug stability is important to consider. The pump refill schedule used in the present study was developed based on the results of a simulated pump stability study. In this stability study, admixtures of $25 \mu \mathrm{g} / \mathrm{mL}$ ziconotide and $35 \mathrm{mg} / \mathrm{mL}$ morphine had an approximately $21 \%$ decline in ziconotide concentration in 17 days. ${ }^{46}$ These results suggest that, during the refill intervals used in the present study ( 1 week for the titration phase and every 2-3 weeks during the extension phase), ziconotide concentrations in patients' pumps could decline considerably. However, stability and its correlation to efficacy were not specifically investigated in the simulated pump stability study or in the present study. Further studies of ziconotide in combination with other opioids are warranted to investigate stability and its correlation with efficacy.

Results from studies in animal models have suggested that ziconotide and morphine may have additive or synergistic effects. In one study, additive analgesic effects of ziconotide and morphine were demonstrated 
with the formalin-induced tonic flinch test; paw pressure, hot plate, and tail immersion tests suggested synergistic effects of ziconotide and morphine. ${ }^{28}$ Animal model studies of the safety of combination ziconotide and morphine therapy have been limited to assessments of respiration and gastrointestinal motility in rats and mice administered IT ziconotide in combination with subcutaneous morphine ${ }^{47}$ and assessments of blood pressure changes in rats administered a combination of IT morphine and ziconotide. ${ }^{48}$ These evaluations suggested that IT ziconotide combined with subcutaneous morphine does not induce respiratory depression and does not increase the susceptibility of morphine-tolerant animals to the respiratory depressant effects of morphine. ${ }^{47}$ However, IT ziconotide does appear to potentiate morphine-induced inhibition of gastrointestinal motility ${ }^{47}$ and to enhance the hypotensive effects of morphine. ${ }^{48}$

The drug combination was useful in multiple chronic pain conditions, including neuropathic pain, mixed pain, and nociceptive pain. Reduced pain intensity was noted as early as week 2 of combination therapy and, by week 5 , the mean decrease in VASPI score was $14.5 \%$ in this small group of patients with highly refractory severe chronic pain. Wallace and colleagues concluded that ziconotide, combined with stable intrathecal morphine, may reduce pain and decrease systemic opioid use in patients with pain inadequately controlled by intrathecal morphine alone; however, they pointed out that clearly, larger, randomized studies with increased duration of follow-up and additional quality of life measures are warranted to investigate the combination of IT ziconotide and IT morphine. ${ }^{45}$

\section{IT ziconotide added to baclofen therapy}

Five of seven patients (71.4\%) had IT ziconotide added to baclofen therapy, for chronic pain. ${ }^{49}$ The mean age was 50.2 years, and the mean baseline VASPI score was $91 \mathrm{~mm}$. All five patients had chronic spasticity and neuropathic pain (mean pain duration, 8.8 years) and had experienced at least one previous failed IT treatment regimen. ${ }^{49}$

The mean dose of ziconotide was $1.7 \mu \mathrm{g}$ /day at initiation and $4.2 \mu \mathrm{g} /$ day at last assessment. ${ }^{49}$ Titration of ziconotide was based on patient response to treatment, and titration details are included in the patient summaries. The mean duration of treatment with ziconotide was approximately 8 months (median duration, 6 months; range, 2-16 months). Intrathecal baclofen was stabilized at a mean dose of 266 mcg/day. ${ }^{49}$ All patients were taking at least 1 systemic opioid during treatment with the study drugs. ${ }^{49}$
Analysis of the primary efficacy measure revealed that VASPI scores improved by a mean of $50.2 \%$ from baseline to last assessment. ${ }^{49}$ The mean time to onset pain relief was 15 weeks (range, 7-29 weeks) with a corresponding mean ziconotide dose of $3.7 \mathrm{mcg} /$ day (range, 1.3-8.1 $\mu \mathrm{g} /$ day). One patient (patient 1) reported AEs (nausea and vomiting with dehydration) during treatment with ziconotide, but the AEs were considered unrelated to ziconotide therapy. ${ }^{49}$

The remaining two patients were receiving ziconotide treatment when baclofen was initiated. Pain intensity scores improved by $75 \%$ and $30 \%$, respectively. Pain relief was evident at two weeks and one week, with corresponding ziconotide doses of $2.4 \mu \mathrm{g} /$ day and $14.4 \mu \mathrm{g} / \mathrm{day}$, respectively. ${ }^{49}$

Studies have shown that baclofen is relatively stable when combined with either morphine $(90 \%$ stable for 30 days) or clonidine ( $90 \%$ stable for $>14$ weeks). ${ }^{50,51}$ On the other hand, the stability of ziconotide tends to be compromised when ziconotide is combined with other agents. ${ }^{45,52,53}$ In an experiment investigating the stability of admixtures containing ziconotide and baclofen, ziconotide maintained $90 \%$ stability for 12 days when combined with a commercially available baclofen solution and for 20 days when combined with powdered baclofen; baclofen concentrations were only minimally affected under the same conditions. ${ }^{54}$

\section{"Intrathecal cocktails"}

Mixtures or combinations of multiple IT analgesic agents are even less well studied than single agents; however, various combinations continue to be utilized in clinical practice and were recognized by the 2007 polyanalgesic consensus conference which reviewed the safety, compatibility, and efficacy data available for various admixtures. ${ }^{43,55}$

The rationale for IT combination therapy is to combine drugs with different mechanisms of action so that afferent pain signals are interrupted at several different sites, thus producing additive or synergistic pain control. This combined effect may also allow for dose reduction of each individual drug, which may reduce drug intolerance.

Ziconotide at concentrations of less than $1 \mathrm{mcg} / \mathrm{mL}$ is not as stable but is very stable at higher concentrations. Morphine and hydromorphone may facilitate ziconotide degradation, thus the 2007 polyanalgesic consensus conference suggestions limiting the concentration and dose of opioid when utilizing opioid/ziconotide admixtures. ${ }^{56}$ Clonidine $2 \mu \mathrm{g} / \mathrm{mL}$ combined with ziconotide is stable (roughly $90 \%$ stable to 60 days) whereas bupivacaine/ziconotide is 
somewhat less stable. ${ }^{52,53} \mathrm{Ziconotide/baclofen} \mathrm{admixtures} \mathrm{are}$ $80 \%$ stable over 30 days (and even more stable when compounded with powdered baclofen).${ }^{54}$ A ziconotide/clonidine/ morphine admixture was $70 \%$ stable for 20 days. ${ }^{53}$

\section{Ziconotide's clinical niche}

Intrathecal ziconotide infusion therapy appears to be a valuable treatment option for moderate to severe pain. Despite being a broad-spectrum analgesic, it seems to be particularly effective in providing pain relief for severe intractable neuropathic pain states, ${ }^{57-59}$ complex regional pain syndromes, ${ }^{59,60}$ and perhaps certain intractable visceral pain states. Its therapeutic niche is still evolving; however, it seems to be especially well suited to be utilized in patients who may not be ideal candidates for opioid therapy. These patient groups may include: patients with active substance abuse issues, patients who are highly sensitive to opioid adverse effects, patients with obstructive sleep apnea, and patients with significant lung disease and/or reduced pulmonary reserve.

\section{Summary}

Ziconotide is a conopeptide intrathecal analgesic which is FDA-approved for the management of severe chronic pain. It is a synthetic equivalent of a naturally occurring conopeptide found in the venom of the fish-eating marine cone snail and provides analgesia via binding to $\mathrm{N}$-type voltage-sensitive calcium channels in the spinal cord.

Ziconotide appears to be potentially beneficial to some patients with intractable pain, but has a narrow therapeutic index. Excessive starting doses initially utilized in early clinical studies performed in the 1990s consistently produced severe dizziness, memory loss, nystagmus, confusion, loss of the ability to word find, and even frank psychosis. ${ }^{36}$ Twelve separate multicenter studies of ziconotide, involving over 1200 patients, spanning more than 10 years, have demonstrated no permanent sequelae of the drug, despite several known instances of inadvertent and profound overdosing. ${ }^{36}$ Multiple studies have shown that used appropriately, ziconotide can be a safe and effective intrathecal analgesic alone or in combination with other intrathecal analgesics. Ziconotide's clinical niche is still evolving but it may be a useful addition to the pharmacologic armamentarium of intrathecal analgesics. Its efficacy as monotherapy, as well as its safe and effective combination with other intrathecal analgesics seem to outweigh its disadvantaged such as its narrow therapeutic window and potentially adverse effects,
It is imperative that the health care provider prescribing ziconotide be familiar with its pharmacology as well as the use of other intrathecal analgesics. The use of a "start low and go slow" approach to dosing/titration of ziconotide remains prudent.

\section{Disclosures}

The authors declare no conflicts of interest.

\section{References}

1. Olivera BM, Rivier J, Clark C, et al. Diversity of Conus neuropeptides. Science. 1990;249(4966):257-263.

2. Olivera BM, Miljanich GP, Ramachandran J, Adams ME. Calciumchannel diversity and neurotransmitter release - the $\omega$-conotoxins and $\omega$-agatoxins. Ann Rev Biochem. 1994;63:823-867.

3. Chung D, Gaur S, Bell JR, Ramachandran J, Nadasdi L. Determination of disulfide bridge pattern in $\omega$-conopeptides. Int J Pept Protein Res. 1995;46(3-4):320-325.

4. Price-Carter M, Hull MS, Goldenberg DP. Roles of individual disulfide bonds in the stability and folding of an $\omega$-conotoxin. Biochemistry. 1998;37(27):9851-9861.

5. Olivera BM. Conus Peptides: Biodiversity-based Discovery and Exogenomics. J Biolog Chem. 2006;281(42):31173-31177.

6. Terlau H, Olivera BM. Conus venoms: a rich source of novel ion channel-targeted peptides. Physiol Rev. 2004;84(1):41-68.

7. Mondal S, Bhavna R, Babu RM, Ramakumar S. Pseudo amino acid composition and multi-class support vector machines approach for conotoxin superfamily classification. J Theor Biol. 2006;243(2):252-260.

8. Lin H, Li QL. Predicting conotoxin superfamily and family by using pseudo amino acid composition and modified Mahalanobis discriminant. Biochem Biophys Res Comm (BBRC). 2007;354:548-551.

9. Teichert RW, Jimenez EC, Twee V, et al. Novel conantokins from Conus parius venom are specific antagonists of N-methyl-D-aspartate receptors. J Biol Chem. 2007;282(51):36905-36913.

10. Khoo KK, Feng ZP, Smith BJ, et al. Structure of the analgesic muconotoxin KIIIA and effects on the structure and function of disulfide deletion (dagger) (double dagger). Biochemistry. 2009. In press.

11. Brose W, Pheifer B, Hassenbusch S, et al. Analgesia produced by SNX-111 in patients with morphine resistant pain. Presented at: 15 th Annual Meeting of the American Pain Society; November 14-17, 1996; Washington, DC.

12. Olivera BM, Cruz LJ, de Santos V, et al. Neuronal calcium channel antagonists: Discrimination between calcium channel subtypes using omegaconotoxin from conus magus venom. Biochemistry. 1987;26(8):2086-2090.

13. Miljanich G, Ramachandran J. Antagonists of neuronal calcium channels: Structure, function, and therapeutic implications. Annu Rev Pharmacol Toxicol. 1995;35:707-734.

14. Staats PS, Yearwood T, Charapata SG, et al. Intrathecal ziconotide in the treatment of refractory pain in patients with cancer or AIDS: A randomized controlled trial. JAMA. 2004;291(1):63-70.

15. Yeager RE, Yoshikami D, Rivier J, Cruz LJ, Miljanich GP. Transmitter release from presynaptic terminals of electric organ: inhibition by the calcium channel antagonist $\omega$ Conus toxin. J Neurosci. 1987;7(8): 2390-2396.

16. Atkinson RA, Kieffer B, Dejaegere A, Sirockin F, Lefevre JF. Structural and dynamic characterization of $\omega$-conotoxin MVIIA: the binding loop exhibits slow conformational exchange. Biochemistry. 2000;39(14):3908-3919.

17. Kim JI, Takahashi M, Ohtake A, Wakamiya A, Sato K. Tyr13 Is essential for the activity of $\omega$-conotoxin MVIIA and GVIA, specific N-type calcium channel blockers. Biochem Biophys Res Commun. 1995;206(2):449-454. 
18. Gohil K, Bell JR, Ramachandran J, Miljanich GP. Neuroanatomical distribution of receptors for a novel voltage-sensitive calcium-channel antagonist, SNX-230 ( $\omega$-conopeptide MVIIC). Brain Res. 1994;653 (1-2):258-266.

19. Bowersox SS, Gadbois T, Singh T, Pettus M, Wang YX, Luther RR. Selective N-type neuronal voltage-sensitive calcium channel blocker, SNX-111, produces spinal antinociception in rat models of acute, persistent and neuropathic pain. J Pharmacol Exp Ther. 1996;279(3):1243-1249.

20. Kristipati R, Nádasdi L, Tarczy-Hornoch K, Lau K, Miljanich GP, Ramachandran J, Bell JR. Characterization of the binding of $\omega$-conopeptides to different classes of non-L-type neuronal calcium channels. Mol Cell Neurosci. 1994;5(3):219-228.

21. Bowersox SS, Luther R. Pharmacotherapeutic potential of omega conotoxin MVIIA (SNX-111), an N-type neuronal calcium channe blocker found in the venom of Conus magus. Toxicon. 1998;36(11): 1651-1658.

22. Wang YX, Pettus M, Gao D, Phillips C, Bowersox SS. Effects of intrathecal administration of ziconotide, a selective neuronal N-type calcium channel blocker, on mechanical allodynia and heat hyperalgesia in a rat model of postoperative pain. Pain. 2000;84(2-3):151-158.

23. Shopp GM, Skov MJ, Yaksh TL. Subchronic toxicity of ziconotide administered by continuous intrathecal infusion in rat and dog. Toxicologist (Suppl to Toxicol Sci). 2004; 78 (S-1): Abstract 2097.

24. Hassenbusch SJ, Portenoy RK, Cousins M, et al. Polyanalgesic Consensus Conference 2003: An update on the management of pain by intraspinal drug delivery-report of an expert panel. J Pain Symptom Manage. 2004;27(6):540-563.

25. Yaksh TL, Horais KA, Tozier NA, et al. Chronically infused intrathecal morphine in dogs. Anesthesiology. 2003;99(1):174-187.

26. Skov MJ, Beck JC, de Kater AW, Shopp GM. Nonclinical safety of ziconotide: an intrathecal analgesic of a new pharmaceutical class. Int J Toxicol. 2007;26(5):411-421.

27. Malmberg AB, Yaksh TL. Effect of continuous intrathecal infusions of mega-conoceptides, N-type calcium-channel blockers, on behavior and antinociception in the formalin and hot-plate tests in rats. Pain. 1995;60(1):83-90.

28. Wang YX, Gao D, Pettus M, Phillips C, Bowersox SS. Interactions of intrathecally administered ziconotide, a selective blocker of neuronal N-type voltage-sensitive calcium channels, with morphine on nociception in rats. Pain. 2000;84(2-3):271-281.

29. Miljanich GP. Ziconotide: neuronal calcium channel blocker for treating severe chronic pain. Curr Med Chem. 2004;11(23):3029-3040.

30. Lyseng-Williamson KA, Perry C. Ziconotide. CNS Drugs. 2006;20(4): 331-341.

31. Wermeling D, Drass M, Ellis D, et al. Pharmacokinetics and pharmacodynamics of intrathecal ziconotide in chronic pain patients. J Clin Pharmacol. 2003;43(6):624-636.

32. Wallace MS. Ziconotide: a new nonopioid intrathecal analgesic for the treatment of chronic pain. Expert Rev Neurother. 2006;6(10): 1423-1428.

33. Rauck RL, Wallace MS, Leong MS, et al. Ziconotide 301 Study Group. A randomized, double-blind, placebo-controlled study of intrathecal ziconotide in adults with severe chronic pain. J Pain Symptom Manage. 2006;31(5):393-406.

34. Newcomb R, Abbruscato TJ, Singh T, Nadasdi L, Davis TP, Milhanich G Bioavailability of ziconotide in brain: influx from blood, stability, and diffusion. Peptides. 2000;21(4):491-501.

35. www.fda.gov/medwatch/SAFETY/2007/Apr_PI/Prialt_PI.pdf

36. Fisher R, Hassenbusch S, Krames E, et al. A consensus statement regarding the present suggested titration for Prialt (ziconotide) (editorial). Neuromodulation. 2005;8(3):153-154.

37. Vandaele SF, Reader TA. Ca (2+)-sensitive and insensitive omegaconotoxin GVIA binding sites in rat brain. Neuroreport. 1994;5(9): 1121-1124.

38. Wallace MS, Charapata SG, Fisher R, et al. Intrathecal ziconotide in the treatment of chronic nonmalignant pain: a randomized, double-blind, placebo-controlled clinical trial. Neuromodulation. 2006;9(2):75-86.
39. Day RO, Williams KM. Open-label extension studies: do they provide meaningful information on the safety of new drugs? Drug Saf. 2007;30(2):93-105.

40. Wallace MS, Rauck R, Fisher R, Charapata SG, Ellis D, Dissanayake S. Intrathecal ziconotide for severe chronic pain: safety and tolerability results of an open-label, long-term trial. Anesth Analg. 2008;106(2): 628-637.

41. Webster LR, Fisher R, Charapata S, Wallace MS. Long-term intrathecal ziconotide for chronic pain: an open-label study. J Pain Symptom Manage. In press 2008.

42. Bennett G, Burchiel K, Buchser E, et al. Clinical guidelines for intraspinal infusion: report of an expert panel. PolyAnalgesic Consensus Conference 2000. J Pain Symptom Manage. 2000;20(2):S37-S43.

43. Deer T, Krames ES, Hassenbusch SJ, et al. Polyanalgesic Consensus Conference 2007: Recommendations for the Management of Pain by Intrathecal (Intraspinal) Drug Delivery: Report of an Interdisciplinary Expert Panel. Neuromodulation. 2007;10(4):300-328.

44. Webster LR, Fakata KL, Charapata S, Fisher R, Mine M. Open-label, multicenter study of combined intrathecal morphine and ziconotide: addition of morphine in patients receiving ziconotide for severe chronic pain. Pain Med. 2008;9(3):282-290.

45. Wallace MS, Kosek PS, Staats P, Fisher R, Schultz DM, Leong M. Phase II, open-label, multicenter study of combined intrathecal morphine and ziconotide: addition of ziconotide in patients receiving intrathecal morphine for severe chronic pain. Pain Med. 2008;9(3): 271-281.

46. Shields D, Montenegro R, Ragusa M. Chemical stability of admixtures combining ziconotide with morphine or hydromorphone during simulated intrathecal administration. Neuromodulation. 2005;8(4): 257-263.

47. Wang YX, Bowersox SS. Analgesic properties of ziconotide, a selective blocker of N-type neuronal calcium channels. CNS Drug Rev. 2000;6(1):1-20.

48. Horvath G, Brodacz B, Holzer-Petsche U. Blood pressure changes after intrathecal co-administration of calcium channel blockers with morphine or clonidine at the spinal level. Naunyn Schmiedebergs Arch Pharmacol. 2002;366(3):270-275.

49. Saulino M, Burton AW, Danyo DA, Frost S, Glanzer J, Solanki DR. Intrathecal ziconotide and baclofen provide pain relief in seven patients with neuropathic and spasticity: case reports. Eur J Phys Rehabil Med. In Press 2009.

50. Sitaram BR, Tsui M, Rawicki HB, Iam S, Sitaram M. Stability and compatibility of intrathecal admixtures containing baclofen and high concentrations of morphine. Int J Pharmaceutics. 1997;153(1): 13-24.

51. Alvarez JC, De Mazancourt P, Chartier-Kastler E, Denys P. Drug stability testing to support clinical feasibility investigations for intrathecal baclofen-clonidine admixture. J Pain Symptom Manage. 2004;28(3):268-272.

52. Shields D, Monenegro R, Aclan J. Chemical stability of admixtures combining ziconotide and bupivacaine during simulated intrathecal administration. Neuromodulation. 2007;10(Suppl 1):1-5.

53. Shields D, Monenegro R. Chemical stability of ziconotide-clondine hydrochloride admixtures with and without morphine sulfate during simulated intrathecal administration. Neuromodulation. 2007;10 (Suppl 1):6-11.

54. Shields D, Monenegro R, Aclan J. Chemical stability of admixtures combining ziconotide with baclofen during simulated intrathecal administration. Neuromodulation. 2007;10(Suppl 1):12-7.

55. Smith HS, Deer TR, Staats PS, Vijay Singh V, Sehgal N, Cordner H Intrathecal Drug Delivery. Pain Physician. 2008; Opioid Special Issue:11(2S): S89-S104.

56. Trissel LA. Trissel's Stability of Compounded Formulations, 2nd edition. APHA Publications, Washington, DC, 2000.

57. Valia-Vera JC, Villanueva VL, Asensio-Samper JM, López-Alarcón MD, de Andrés JA. Ziconotide: an innovative alternative for intense chronic neuropathic pain. Rev Neurol. 2007;45(11):665-669. 
58. Taira T. Chronix intrathecal drug administration for the control of intractable pain. Brain Nerve. 2008;60(5):509-517.

59. Wermeling DP, Berger JR. Ziconotide infusion for severe chronic pain: case series of patients with neuropathic pain. Pharmacotherapy. 2006;26(3):395-402.
60. Stanton-Hicks M, Kapural L. An effective treatment of severe complex regional pain syndrome type 1 in a child using high doses of intrathecal ziconotide. J Pain Symptom Manage. 2006;32(6):509-511.

\section{Publish your work in this journal}

Therapeutics and Clinical Risk Management is an international, peerreviewed journal of clinical therapeutics and risk management, focusing on concise rapid reporting of clinical studies in all therapeutic areas, outcomes, safety, and programs for the effective, safe, and sustained use of medicines. This journal is indexed on PubMed Central, CAS,
EMBase, Scopus and the Elsevier Bibliographic databases. The manuscript management system is completely online and includes a very quick and fair peer-review system, which is all easy to use. Visit http://www.dovepress.com/testimonials.php to read real quotes from published authors.

Submit your manuscript here: http://www.dovepress.com/therapeutics-and-clinical-risk-management-journal 